Novembre 1991

INFORMATION, STABILITE DES PRIX ET BIEN-ETRE

$$
\text { Bruno JULLIEN }{ }^{*}
$$

$\mathrm{N}^{\circ} 9129$

*ENSAE-CREST et CEPREMAP 


\section{Information, stabilité des prix et bien-être}

\section{RESUME}

Dans le contexte d'une économie stationnaire avec titres réels de durée de vie infinie et ouverture périodique des marchés financiers, nous étudions l'impact sur le bien-être des agents de la structure d'information. A chaque période les agents reçoivent un signal corrélé avec les dividendes futurs des actifs. Lorsque la structure d'information s'affine (du fait par exemple de la présence d'"initiés"), la volatilité des prix augmente. Les dividendes des actifs sont mieux prédits mais le risque en capital augmente, puisque les prix futurs deviennent moins prédictibles. Le risque global sur l'investissement des agents peut alors augmenter. Le papier exhibe un exemple où, en raison de cet effet, l'absence de toute information domine toute autre structure.

Mots clefs: Information, volatilité des prix, risque, bien-être.

Codes J.E.L.: $026,313$.

\section{Information, Price Stability and Welfare}

\section{ABSTRACT}

In the context of a stationary economy, where markets for long-lived real assets open periodically, we analyse the welfare effects of the information structure. At every period, agents receive a signal related to the future dividends of assets. Whenever the quality of the information increases, the price volatility increases. Assets dividends are more predictable but at the expense of a higher capital risk, since future prices are less predictable. The global risk on assets returns may then increase, resulting in a lower welfare level. The paper exhibits an example where, due to this effect, agents prefer a structure where nobody receives any information to any other stationary information structure.

Keywords: Information, price volatility, risk, welfare.

J.E.L. Classification Numbers: 026,313 . 


\section{INTRODUCTION}

Faut-il interdire les "délits d'initiés", e.g. les transactions boursières effectuées par des agents privés ou des institutions sur la base d'informations privilégiées, comme c'est actuellement le cas dans de nombreux pays et en particulier en France? Cette question reste encore de nos jours un point de controverse à la fois dans la littérature juridique et dans la littérature économique. Le problème est complexe et du point de vue économique comporte trois aspects majeurs: comment sont affectés les capacités de financement sur les marchés boursiers des entreprises lorsque les agents anticipent que certains initiés vont participer aux échanges futurs de titres? Dans quel mesure un agent informé peut-il manipuler le marché au détriment des autres participants? Finalement, en l'absence de tout comportement stratégique visant à exploiter un pouvoir de marché, comment évolue le bien-être des intervenants lorsque certains d'entre eux disposent d'une information plus fine? Ce papier s'intéresse exclusivement à ce troisième aspect.

Un argument commun en faveur de l'autorisation des opérations d'initiés est que l'information privée de ces agents sera au moins partiellement révélée par les prix aux autres intervenants. Ceux-ci disposant alors d'une meilleure information seront à même de choisir leurs portefeuilles d'actifs de façon plus efficace et de réduire le risque sur leurs placements. On peut parler ici d'une augmentation de l'efficacité interim (après que les agents aient obtenu l'information privée). Hirshleifer [1971] a montré que les choses ne sont pas si simple. Si l'on se place du point de vue d'un participant potentiel au marché avant que celui-ci n'ouvre (ex-ante), une plus grande information révélée par les prix résulte en un accroissement de l'incertitude sur les prix auquels les échanges auront lieu et donc à un plus grand risque sur les termes de l'échange. Ceci peut aboutir à une 
baisse du bien-être collectif.

De façon générale, le problème s'inscrit dans le cadre plus général de l'analyse normative des équilibres en anticipations rationnelles avec information asymétrique (voir Laffont [1985]). Les économistes ont cependant développé des modèles spécifiques et maniables pour l'études des marchés financiers (voir en particulier Grossman-Stiglitz [1980] et Kyle [1985]). Ces modèles ont été exploités en vue de fournir une évaluation de l' "externalité" que des agents informés exercent sur les autres participants au marché (Leland [1990], Bajeux-Rochet [1988]). Ces papiers mettent en particulier en regard les gains d'efficacité allocative interim et l'augmentation du risque ex-ante. Les résultats restent cependant ambigus et très liés au choix de modélisation. Le présent papier essaie de développer quelques remarques concernant la façon d'aborder le problème.

Du fait de la nature des modèles existants, les papiers mentionnés ci-dessus sont essentiellement basés sur des arguments statiques, ou du moins de court terme. Le marché n'ouvre qu' une fois et le revenu futur des actifs est exogène. Si l'on se place d'un point de vue dynamique avec ouverture régulière des marchés pour des actifs à longue maturité, ce n'est plus le cas puisque les gains en capitaux sur les différents actifs dépendent des prix d'équilibre futurs qui sont endogènes. Dans un tel contexte deux points apparaissent rapidement. Le premier point est immédiat. Dans un cadre de marchés incomplets les tranferts intertemporels seront en général inefficaces. La structure d'information affecte alors l'efficacité intertemporelle de l'allocation d'équilibre de façon à priori ambiguë, et il convient de déterminer dans quel sens va cette effet. Le second point est celui sur lequel nous voulons insister ici.

Dans un contexte d'ouverture régulière des marchés, la présomption selon laquelle lorsque l'information révélée aux agents devient plus fine 
ceux-ci disposent d'une "meilleure" information leur permettant de diminuer le risque sur leurs investissements est fausse. Considérons par exemple un marché boursier. Sur ce marché un agent privé détermine ses échanges d'actions de différentes entreprises en fonction de son évaluation conjointe des dividendes futurs et des prix futurs des titres. Sur un marché où très peu d'information est disponible à chaque période, les prix restent relativement stables et sont donc facile à prévoir. Par contre si à chaque période les individus disposent d'une information très précise sur les dividendes futurs, les prix vont réagir à cette information et devenir très volatiles. Comme les prix futurs dépendent de l'information future et non de l'information courrante, ils seront alors très difficiles à prévoir. En d'autres termes accroitre l'information sur les dividendes n'aboutit pas automatiquement à une meilleurs information sur la rentabilité des actifs, mais à un déplacement de l'incertitude des dividendes vers les prix. Le papier montre que l'effet de déstabilisation des prix peut être suffisant pour aboutir à une valeur sociale négative de l'information. De fait nous contruisons une économie telle que, comparée à la situation où aucune information n'est disponible, toute structure stationnaire d'information a une valeur sociale négative.

Le modèle est un modèle à générations imbriquées avec titres réels et un agent représentatif, dont nous étudions les équilibres stationnaires. Dans cette économie, le portefeuille d'actif détenu par l'agent est fixe et égal au portefeuille aggrégé. De ce fait il n'existe pas de possibilités d'assurance autres que celles portant sur les mouvements de prix et les gains en plue-value. L'utilité espérée d'un agent dépend de la valeur moyenne du portefeuille de marché, et de la volatilité des prix. Nous montrerons que même si la variation de la valeur moyenne du portefeuille aggrégé a un effet positif sur l'utilité lorsque l'information augmente, cet 
effet est toujours compensé par la déstabilisation des prix.

\section{Le modèle}

Considérons une économie stationnaire avec un bien périmable et des générations imbriquées vivant deux périodes. Chaque génération est composée d'un continuum (de masse 1) d'agents identiques. Chaque individu détient $e$ unités de bien à la naissance et n'a pas de ressources pendant sa seconde période de vie. Lorsqu' un agent consomme $c_{1}$ et $c_{2}$ durant les deux périodes de sa vie, il obtient une utilité (au sens de von Neumann-Morgeinstein):

$$
U\left(c_{1}, c_{2}\right)=c_{1}+v\left(c_{2}\right)
$$

Hypothèse $0: \quad v($.$) est croissante, concave et v^{\prime}(c)<1$ pour $c$ assez élevé,

$$
\begin{aligned}
& c v^{\prime}(c) \text { est croissante, } \\
& v(c)-c v^{\prime}(c) \text { est concave, }
\end{aligned}
$$

La première partie de l'hypothèse garantie l'existance d'un équilibre. La seconde partie postule que les consommations de première période et de seconde période sont des substituts. Elle implique que le prix d'équilibre d'un titre croit lorsque le revenu total du titre croit. Cette condition est standard dans les modèle à générations, elle correspond à l'idée que l'épargne croit si le taux d'intérêt croit. Si $r(c)=-c v^{\prime \prime}(c) / v^{\prime}(c)$ dénote l'indice relatif d'aversion pour le risque, la condition est équivalente à $r(c) \leq 1$. La dernière condition est plus spécifique. Dans un modèle sans aléa cette condition implique que l'utilité obtenue en équilibre par un agent est une fonction concave de sa consommation de deuxième période. Elle est équivalente à $c r^{\prime}(c) / r(c) \leq r(c)$, il faut donc que $r(c)$ ne croisse pas trop vite.

L'économie évolue de façon stochastique. A chaque date, elle peut être dans I états différents: 


$$
s_{t} \in\left\{s_{1}, \ldots \ldots, s_{1}\right\}
$$

où $s_{t}$ suit un processus de Markov stationnaire.

Il existe $J$ titres réels de durée de vie infinie, chacun en quantité totale 1. Lorsque l'état de l'économie est $s_{i}$, le titre $j$ rapporte un dividende en bien $R_{j}\left(s_{i}\right)$. Nous désignons par $\mathbf{R}(s)$ le vecteur de production dans l'état $s$ :

$$
R(s)=\left\{R_{1}(s), \ldots \ldots, R_{J}(s)\right\}^{\prime} .
$$

Finallement nous désignons par $D(s)$ la production totale dans l'état $s$ :

$$
D(s)=\sum_{j=1}^{\mathrm{J}} R_{\mathrm{j}}(s) \text {. }
$$

Les états sont indexés de façon à ce que la production totale croisse avec l'état: $D\left(s_{i+1}\right) \geq D\left(s_{i}\right)$. Dans la situation de départ l'état $s_{t}$ peut être connu à la date $t$ par la vieille génération mais n'est pas observé par la jeune génération.

Nous nous intéressons uniquement à des équilibres intérieurs où les agents consomment des quantités positives durant les deux périodes de leur vie. Un équilibre stationnaire est caractérisé par un vecteur de prix unique $\overline{\mathbf{p}}=\left\{\bar{p}_{j}\right\}$, vérifiant:

$$
\begin{aligned}
& \overline{\mathbf{p}}=\mathrm{E}\left\{v^{\prime}\left(D\left(s_{\mathrm{t}+1}\right)+\bar{q}\right)\left(\mathbf{R}\left(s_{t+1}\right)+\overline{\mathbf{p}}\right)\right\}, \\
& \bar{q}=\sum_{j=1}^{\mathrm{J}} \bar{p}_{\mathrm{j}}<e .
\end{aligned}
$$

Notez que le prix ne révèle aucune information puisque l'offre de titres de la vieille génération est fixe. La première équation égalise l'utilité marginale espérée du revenu de chaque titre à son coût pour une quantité 1 de titre. La deuxième inégalité garantie que l'équilibre est intérieur. Le prix $\bar{q}$ représente la valeur totale du portefeuille de marché. L'évolution de cette valeur totale sera au coeur de l'analyse. L'existence d'un tel équilibre découle d'arguments standards. 
Proposition 1: Si e est assez grand, il existe un équilibre stationnaire intérieur $\overline{\mathbf{p}}$.

Preuve: voir annexe.

Considérons l'utilité espérée ex-ante par un individu à l'équilibre stationnaire. Un calcul aisé montre que sa valeur est:

$$
\bar{W}=e-\bar{q}+E\left\{v\left(D\left(s_{t}\right)+\bar{q}\right)\right\}
$$

où l'espérance est prise par rapport à la loi inconditionnelle du processus. Ceci appelle un premier commentaire sur l'équilibre. Si l'on considère les allocations obtenues à partir d'un transfert fixe $x$ de la jeune génération vers la vieille génération, l'utilité espérée des agents est $e-x+E\left\{v\left(D\left(s_{t}\right)+x\right)\right\}$. Parmi ces allocations, l'allocation optimale est obtenue pour un transfert vérifiant $1=E\left\{v^{\prime}\left(D\left(s_{t}\right)+x\right)\right\}$. L'équilibre de l'économie est obtenu à un niveau de transfert sur-optimal: $1>E\left\{v^{\prime}\left(D\left(s_{t}\right)+\bar{q}\right)\right\}$. Le prix total est trop élevé (le taux d'intérêt sans risque, défini à la marge, est positif alors qu'il devrait être nul puisqu' il n'y a pas croissance de la population).

\section{L'économie avec information}

Supposons que, à chaque date $t$, une fraction $\varepsilon$ de la jeune génération observe un signal $\phi_{t} \in\left\{\phi_{1}, \ldots \ldots, \phi_{L}\right\}$ corrélé avec les états futurs $s_{t+1}, \ldots$ Les signaux sont classés: $\phi_{j} \leq \phi_{j+1}$. L'hypothèse générale est que $\left\{s_{t}, \phi_{t}\right\}$ suit un processus stochastique de Markov stationnaire. L'état $s_{t}$ peut être connu à la date $t$ par la jeune génération si il est inclus dans le signal $\phi_{t}$. Nous voulons modéliser une situation où les dividendes aggrégés sont positivement corrélés dans le temps et où nous pouvons classer l'information depuis la plus mauvaise nouvelle vers la meilleure nouvelle, une bonne nouvelle ayant naturellement plus de chance d'être observée après un bon état. Pour ceci nous supposons: 
Hypothèse 1: La loi de $s_{t+1}$ conditionnelle à $\phi_{t}=\phi_{j+1}$ domine stochastiquement au premier ordre la loi de $s_{t+1}$ conditionnelle à $\phi_{t}=\phi_{j}$. La loi de $\phi_{t}$ conditionnelle à $s_{t}=s_{1+1}$ domine stochastiquement au premier ordre la loi de $\phi_{t}$ conditionnelle à $s_{t}=s_{i}$.

Pour fixer les idées on pourra penser au cas simple où le signal consiste en fait dans l'observation de l'état courrant: $\phi_{t}=s_{t}$. Dans ce cas l'hypothèse revient à supposer que la loi du dividende total futur conditionnelle au dividende total courrant croit avec le dividende courrant (toujours au sens de la dominance stochastique du premier ordre) ${ }^{1}$.

Considérons un équilibre stationnaire de cette économie. Comme le signal est à valeurs discrètes, l'équilibre sera génériquement totalement révélateur, e.g. la valeur du signal peut être déduite de la valeur des prix. Nous supposons donc que c'est le cas ici, de sorte que tous les membres de la génération née en $t$ connaissent $\phi_{t}$ à l'équilibre. Un équilibre stationnaire pour cette économie est caractérisé par un vecteur de prix $p(\phi)=\left\{p_{j}(\phi)\right\}_{j}$ pour les titres (exprimés en unités de bien) tel que la demande de chaque titre soit 1 . Un tel équilibre est donné par les conditions:

$$
\begin{aligned}
& \mathbf{p}\left(\phi_{t}\right)=E\left\{v^{\prime}\left(D\left(s_{t+1}\right)+q\left(\phi_{t+1}\right)\right)\left(R\left(s_{t+1}\right)+p\left(\phi_{t+1}\right)\right) \mid \phi_{t}\right\}, \\
& q\left(\phi_{t}\right)=\sum_{j=1}^{J} p_{j}\left(\phi_{t}\right)<e .
\end{aligned}
$$

Le prix $q\left(\phi_{t}\right)$ représente la valeur totale du portefeuille de marché lorsque l'information de la jeune génération est $\phi_{t}$. Nous serons plus

1 Les résultats du papier sont aussi valables si il n'y a pas de risque aggrégé $(D(s) \equiv D)$. 
particulièrement intéressés par la valeur espérée inconditionnelle du portefeuille de marché:

$$
q=E\left\{q\left(\phi_{t}\right)\right\}
$$

Proposition 2: Si e est assez grand, il existe un équilibre stationnaire intérieur $\bar{p}\left(\phi_{t}\right)$ qui vérifie:

$$
E\left\{q\left(\phi_{t}\right) \mid s_{t}=s_{1+1}\right\} \geq E\left\{q\left(\Phi_{t}\right) \mid s_{t}=s_{i}\right\}
$$

Preuve: voir annexe.

Pour cette économie, l'utilité espérée d'un individu en équilibre stationnaire est donnée par:

$$
W=e-q+E\left\{v\left(D\left(s_{t}\right)+q\left(\phi_{t}\right)\right)\right\}
$$

Nous sélectionnons un équilibre pour chaque économie et comparons le niveau d'utilité espéré ex-ante par un agent dans chacun des équilibres. Notez que l'équilibre peut ne pas être unique. L'analyse est alors valide pour toute selection pour peu que l'équilibre dans l'économie avec signal vérifie la propriété de la proposition 2.

\section{Structure d'information optimale.}

Le but de cette section est donc de comparer $W$ et $\bar{W}$. On peut voir la situation comme suit. La jeune génération choisit la structure d'information en début de période $t$ avant que le signal ne soit généré (sous l'hypothèse que l'équilibre s'établit immédiatement à l'équilibre stationnaire). En d'autres termes, les opérations d'initiés sont initiallement interdites et la génération considère la possibilité de les autoriser. La vielle génération à la date $t$ évalue alors le changement à l'aide de $E\left\{v\left(D\left(s_{t}\right)+q\left(\phi_{t}\right)\right)\right\}$ (avant de connaitre l'état $s_{t}$ ) de sorte que si $q<\bar{q}$, elle préfère la structure sans information (le cas inverse est 
ambigu).

On peut interpréter le passage de $\bar{W}$ à $W$ en deux temps. Dans un premier temps la valeur totale du portefeullle de marché passe de $\bar{q}$ à $q$, modifiant ainsi la valeur moyenne du tranfert intergénérationnel. Dans un deuxième temps la volatilité des prix augmente du prix certain $q$ au prix incertain $q\left(\phi_{t}\right)$. A ce stade il convient de revenir sur les hypothèses de départ. Nous avons supposé que l'utilité était linéaire en consommation de première période. Celà permet de bien isoler les deux effets recherchés. En particulier un agent ne subit aucune perte d'utilité du fait de la volatilité des prix de première période, comme ce serait le cas dans les modèles à 1 période traditionnellement utilisés pour l'analyse des marchés financiers. Les seuls effets à l'oeuvre du point de vue d'un agent lorsque l'on passe de l'équilibre $\overline{\mathbf{p}}$ à l'équilibre $\mathbf{p}\left(\phi_{t}\right)$ sont une variation du prix moyen, et une augmentation de la volatilité des prix futurs. L'idée générale est que les fluctuations de prix résultent dans un accroissement du risque. Ceci n'est évidemment pas toujours le cas. En effet il se peut que les mouvements de prix fournissent de l'assurance contre les fluctuations de dividendes (si la corrélation entre les deux est négative). Ceci semble cependant irréaliste et ce n'est effectivement pas le cas dans notre modèle.

Lemme 1: $W<e-q+E\left\{v\left(D\left(s_{t}\right)+q\right)\right\}$.

Preuve: $\quad W=e-q+E\left\{E\left\{v\left(D\left(s_{t}\right)+q\left(\phi_{t}\right)\right) \mid s_{t}\right\}\right\}$ et donc $W \leq e-q+E\left\{v\left(D\left(s_{t}\right)+E\left\{q\left(\phi_{t}\right) \mid s_{t}\right\}\right)\right\}$.

D'après la proposition 2, la variable aléatoire $D\left(s_{t}\right)+q$ domine la variable $D\left(s_{t}\right)+E\left\{q\left(\phi_{t}\right) \mid s_{t}\right\}$ au sens de la dominance stochastique du deuxième ordre. Comme $v($.$) est concave le résultat s'ensuit.$

Q.E.D. 
Le lemme exprime bien l'idée que les fluctuations de prix ne fournissent pas d'assurance, puisqu'une stabilisation au niveau moyen augmente l'utilité. A cet effet de déstabilisation il faut rajoutter la variation de prix moyen. Ce second effet peut être positif ou négatif. Etant donné que le prix $\bar{q}$ est déjà sur-optimal dans la classe des allocations obtenues par un tranfert fixe d'une génération à l'autre, il est clair que la variation de prix aura un effet négatif sur l'utilité si le prix croit, positif si il décroit. De ce fait on obtient immédiatement:

Proposition 3: Si $q \geq \bar{q}$ alors $W<\bar{W}$.

Preuve: $f(x)=e-x+E\left\{v\left(D\left(s_{t}\right)+x\right)\right\}$ est concave en $x$ et sa pente est $f^{\prime}(x)=E\left\{v^{\prime}\left(D\left(s_{t}\right)+x\right)\right\}-1$. Comme $\bar{q}=E\left\{v^{\prime}\left(D\left(s_{t}\right)+\bar{q}\right)\left(D\left(s_{t}\right)+\bar{q}\right)\right\}, \quad$ la pente en $x=\bar{q}$ est négative de sorte que $f(q) \leq f(\bar{q})$.

Q.E.D.

Il faut cependant remarquer que lorsque $q>\bar{q}$, l'équilibre de l'économie sans signal ne domine pas à priori l'équilibre de l'économie avec signal puisque la vieille génération bénéficie d'une consommation plus élevée dans cette dernière. De plus le résultat est moins du à la déstabilisation des prix qu'à la sur-optimalité du prix total. Le cas inverse nous intéresse donc plus.

Proposition 4: Si $q<\bar{q}$ alors $W<\bar{W}$.

Preuve: D'après la condition d'équilibre:

$q=E\left\{v^{\prime}\left(D\left(s_{t}\right)+q\left(\phi_{t}\right)\right)\left(D\left(s_{t}\right)+q\left(\phi_{t}\right)\right)\right\}$, et donc

$W=e+E\left\{v\left(D\left(s_{t}\right)+q\left(\phi_{t}\right)\right)-v^{\prime}\left(D\left(s_{t}\right)+q\left(\phi_{t}\right)\right)\left(D\left(s_{t}\right)+q\left(\phi_{t}\right)\right)\right\}$.

Pour les raisons avancées dans la preuve du lemme 1 , comme 
$v(c)-c v^{\prime}(c)$ est concave:

$W<g(q)=e+E\left\{v\left(D\left(s_{t}\right)+q\right)-v^{\prime}\left(D\left(s_{t}\right)+q\right)\left(D\left(s_{t}\right)+q\right)\right\}$, tandis que $\bar{W}=g(\bar{q})$. Mais la fonction $g($.$) est croissante (puisque v($. est concave).

Q.E.D.

Dans le cadre de la proposition 4, la variation du prix moyen a un effet positif sur le bien-être des agents. Cependant cet effet est toujours contrebalancé par l'augmentation de la volatilité des prix futurs. Le résultat dépend fortement de la linéarité de $U\left(c_{1}, c_{2}\right)$ en $c_{1}$. Abandonner cette hypothèse nous obligerait à considérer l'effet de la variabilité des prix sur un agent au cours de la première période de sa vie, effet qui est beaucoup plus ambigu que celui mis en avant ici.

\section{Un exemple:}

Considérons le cas d'une fonction d'utilité $v($.$) ayant un indice$ relatif d'aversion pour le risque constant:

$$
v(x)=\delta \frac{x^{1-\sigma}}{1-\sigma} \text { avec } 1>\sigma>0
$$

L'hypothèse 0 est bien vérifiée et les proposition 3 et 4 sont valables. La condition d'équilibre s'écrit:

$$
q=E\left\{\delta\left(D\left(s_{t}\right)+q\left(\phi_{t}\right)\right)^{1-\sigma}\right\} .
$$

A partir de cette relation, on peut réécrire le bien être d'un agent de la façon suivante:

$$
\begin{aligned}
& W=e+\frac{\sigma}{1-\sigma} q \\
& \bar{W}=e+\frac{\sigma}{1-\sigma} \bar{q}
\end{aligned}
$$

On obtient donc comme corollaire immédiat:

Corollaire: Si $v($.$) a un indice relatif d'aversion pour le risque constant$ et inférieur à 1 , alors: 


$$
w<\bar{W} \text {, et } q<\bar{q} \text {. }
$$

Nous pouvons donc dans ce cas prédire le mouvement des prix: le prix moyen baisse si les agent n'ont pas trop d'aversion pour le risque. Ce cas est particulièrement intéressant puisqu'alors en début de période $t$, la vieille génération et la jeune génération seront d'accord pour préférer l'absence d'information.

\section{Conclusion}

Le papier s'est attaché à mettre en évidence comment une information trop abondante peut s'avérer nuisible en introduisant trop d'incertitude sur les rendements futurs des actifs en raison de la forte volatilité des gains en capitaux, même si l'effet sur le niveau des prix est positif. Pour ce faire nous avons choisi un cadre où les agents présents sur un marché ne disposent d'aucune possibilité d'assurance. L'analyse apparait donc complémentaire de l'analyse des problèmes d'information en terme de destruction de marchés d'assurance potentiels, comme dans Hirshleifer [1979]. L'analyse apporte quelques clarifications sur le problème des délits d'initiés. Elle suggère qu'un aspect complémentaire de la manipulation stratégique des prix par des agents disposant d'informations privilégiées (voir Kyle [1985]) est le risque en capital introduit par la révélation de cette information sur le marché. Le bon niveau de transparence des marchés peut alors résulter d'un compromis entre la prévention des manipulations et la stabilité des prix. 


\section{Références}

Bajeux I. et J.C. Rochet [1991], "Opérations d'initiés: une analyse de surplus", Finance.

Grossman S.J. et J.E. Stiglitz [1980], "On the Impossibility of Informationally Efficient Markets", American Economic Review, vol.70, n³, 393-408.

Hirshleifer J. [1971], "The Private and Social value of Information and the Reward to Inventive Activity", American Economic Review, 561-574.

Kyle A. [1985], "Continuous Auctions and Insider Trading", Econometrica 53, 1315-1336.

Laffont J.J. [1985], "On the Welfare Analysis of Rational Expectations Equilibria with Asymmetric Information", vol.53, $n^{\circ} 1,1-29$.

Leland H.E. [1990], "Insider Trading: Should It Be Prohibeted", Mimeo 


\section{Annexe}

Preuve des propositions 1 et 2 .

Nous prouvons la proposition 2, la preuve de la proposition 1 est identique. Considérons la fonction de $[0, e]^{\mathrm{JL}}$ dans $\mathbb{R}^{\mathrm{JL}}$ qui à $\mathbf{P}=$ $\left\{p_{j}\left(\phi_{1}\right)\right\}_{j 1}$ associe $\psi(P)=\left\{\tilde{p}_{j}\left(\phi_{1}\right)\right\}_{j 1}$ défini par:

$$
\tilde{p}_{j}\left(\phi_{1}\right)=E\left\{v^{\prime}\left(D\left(s_{t+1}\right)+\sum_{k} p_{k}\left(\phi_{t+1}\right)\right)\left(R_{j}\left(s_{t+1}\right)+p_{j}\left(\phi_{t+1}\right)\right) \mid \phi_{t}=\phi_{1}\right\},
$$

Soit $R=\sup R_{j}\left(s_{i}\right)$ et $r=\inf R_{j}\left(s_{1}\right)$, alors

$0<\tilde{p}_{j}\left(\phi_{1}\right)<\sup _{r<x<\mathrm{R}+\mathrm{e}} V^{\prime}((\mathrm{J}-1) r+x) x<e$

si $e$ est assez grand puisque $v^{\prime}(c)<1$ pour $c$ grand. Donc $\psi$ admet un point fixe qui est un équilibre intérieur.

Le prix du portefeuille de marché vérifie:

$$
q\left(\phi_{1}\right)=E\left\{v^{\prime}\left(D\left(s_{t+1}\right)+q\left(\phi_{t+1}\right)\right)\left(D\left(s_{t+1}\right)+q\left(\phi_{t+1}\right)\right) \mid \phi_{t}=\phi_{1}\right\}
$$

Il s'agit ici du point fixe d'une fonction $\chi$ sur $[0, e]^{\mathrm{L}}$ définie comme précédemment. Considérons deux vecteurs $q=\left\{q\left(\phi_{1}\right)\right\}$ tel que $q\left(\phi_{1+1}\right) \geq$ $q\left(\phi_{1}\right)$, alors la loi de $D\left(s_{t+1}\right)+q\left(\phi_{t+1}\right)$ conditionnelle à $\phi_{t}=\phi_{1+1}$ domine la loi de $D\left(s_{t+1}\right)+q\left(\phi_{t+1}\right)$ conditionnelle à $\phi_{t}=\phi_{1}$. Comme $v^{\prime}(c) c$ est croissante, l'image $\mathbf{q}^{\prime}=\chi(\mathbf{q})$ vérifie aussi la propriété $q^{\prime}\left(\phi_{1+1}\right) \geq q^{\prime}\left(\phi_{1}\right)$. La propiété est donc vérifiée pour au moins un point fixe.

Q.E.D. 